Portland State University

PDXScholar

$1-1-1982$

\title{
Existence of the Dielectric Constant in Fluids of Classical Deformable Molecules
}

John D. Ramshaw

Portland State University, jdramshaw@yahoo.com

Follow this and additional works at: https://pdxscholar.library.pdx.edu/phy_fac

Part of the Physics Commons

Let us know how access to this document benefits you.

Citation Details

J.D. Ramshaw, "Existence of the dielectric constant in fluids of classical deformable molecules," J. Chem. Phys. 76, 2635 (1982)

This Article is brought to you for free and open access. It has been accepted for inclusion in Physics Faculty Publications and Presentations by an authorized administrator of PDXScholar. Please contact us if we can make this document more accessible: pdxscholar@pdx.edu. 


\title{
Existence of the dielectric constant in fluids of classical deformable molecules ${ }^{\text {a) }}$
}

\author{
John D. Ramshaw \\ Theoretical Division, University of California, Los Alamos National Laboratory, Los Alamos, New \\ Mexico 87545 \\ (Received 29 September 1981; accepted 13 November 1981)
}

\begin{abstract}
The existence of the dielectric constant $\epsilon$ is investigated for fluids composed of classical deformable (polarizable) molecules. The development is based upon generalized functional-derivative relations which involve joint distributions in molecular positions $r_{k}$ and dipole moments $\mu_{k}$. Sufficient conditions for the existence of $\epsilon$ are expressed in terms of the generalized direct correlation function $c(12)=c\left(\mathbf{r}_{1}, \mu_{1} ; \mathbf{r}_{2}, \mu_{2}\right)$. It is found that $\epsilon$ exists if $-k T c(12)$ depends only on relative positions and dipole moment directions (in addition to $\left|\mu_{1}\right|$ and $\left.\left|\mu_{2}\right|\right)$, and becomes asymptotic to the dipole-dipole potential at long range. An expression for $\epsilon$ in terms of a short-ranged total correlation function $h_{0}(12)$ emerges automatically from the development. An expression for $\epsilon$ in terms of $c(12)$ is also derived. The latter expression involves an inverse kernel in $\left(\left|\mu_{1}\right|,\left|\mu_{2}\right|\right)$ space. The case of rigid polar molecules is reconsidered as a special case of the present formulation.
\end{abstract}

\section{INTRODUCTION AND SUMMARY}

The molecular theory of dielectric fluids has experienced something of a renaissance in recent years. ${ }^{1-4}$ Much of this recent work has been restricted to rigid (unpolarizable) polar molecules, which are much simpler to deal with than are polarizable molecules. This restriction has greatly facilitated the clarification of certain subtle issues related to the long-range nature of dipolar interactions. Now that a satisfactory under standing of these issues has been achieved, a greater emphasis is being placed on polarizable molecules. ${ }^{3-10}$ This emphasis is welcome and necessary; all real molecules are polarizable, and the effects of polarizability are quantitatively significant even for molecules with large permanent dipole moments. Inclusion of polar izability is therefore essential for the quantitative inter pretation of experimental data.

Polarizability is associated with internal (vibrational and electronic) molecular degrees of freedom, whose proper treatment requires the use of quantum mechanics. Unfortunately, such a treatment entails a vast increase in the complexity of the problem. Even when the polar izability is regarded as a fixed parameter (which is a rather severe idealization), an esoteric graphical formalism of formidable complexity is needed. ${ }^{3}$ However, there is a growing realization that classical models of the internal degrees of freedom are both sensible and useful, ${ }^{6-10}$ even though they are not faithful to the underlying physics. The model parameters (e.g., restoring force constants) can be chosen so that the classical behavior mimics the true quantum behavior in most essential respects. The advantage of a purely classical description is its simplicity; the internal coordinates can be lumped together with molecular positions and orientations, and the resulting description is hardly more complicated than that for rigid molecules. The main disadvantage of a purely classical treatment is that it yields incorrect dispersion forces between the molecules, $7,8,11$ but this difficulty may be overcome simply by including

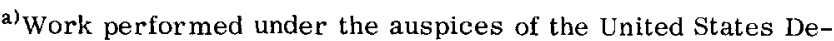
partment of Energy.
}

an artificial compensating term in the intermolecular potential. ${ }^{7}$

The main purpose of the present article is to establish sufficient conditions for the existence of the dielectric constant $\epsilon$ in fluid systems of purely classical deformable (polarizable) molecules. Aside from the requirement that internal degrees of freedom be treated classically, there is no restriction on the molecular model or internal molecular structure. Our development constitutes the extension to classical deformable molecules of previous work on rigid polar molecules. ${ }^{12}$ It is based upon generalized functional-derivative relations which involve joint distributions in molecular positions $\mathbf{r}_{k}$ and dipole moments $\mu_{k}$. These relations give rise to a generalized direct correlation function $c(12)=c\left(\mathbf{r}_{1}, \mu_{1} ; \mathbf{r}_{2}, \boldsymbol{\mu}_{2}\right)$, in terms of which sufficient conditions for the existence of $\epsilon$ can be simply expressed. It is found that $\epsilon$ exists if $c(12)$ depends only on relative positions and dipole moment directions (in addition to $\left|\mu_{1}\right|$ and $\left.\left|\mu_{2}\right|\right)$, and becomes asymptotic to $-\theta(12) / k T$ at long range, where $\theta(12)$ is the dipole-dipole potential. These conditions are straightforward generalizations of the sufficient conditions for the existence of $\epsilon$ in fluids of rigid polar molecules. ${ }^{12}$

In the usual way, an expression for $\epsilon$ in terms of a short-ranged total correlation function $h_{0}(12)$ emerges automatically from the development. This expression has not previously been presented, although it is closely related to the known expression for $\epsilon$ in fluid mixtures of rigid polar molecules. ${ }^{13,14}$ (H $\phi y$ ye and Stell ${ }^{8,9}$ have systematically exploited the correspondence between fluids of deformable molecules and fluid mixtures of rigid molecules.) A new expression for $\epsilon$ in terms of $c(12)$ is also derived. This expression involves an inverse kernel in $\left(\left|\mu_{1}\right|,\left|\mu_{2}\right|\right)$ space, which is analogous to the inverse matrices that occur in the corresponding expressions for rigid polar molecules. ${ }^{12,14}$

Finally, fluids of rigid polar molecules are briefly reconsidered as a special case of the present formulation. When this specialization is performed, it is found that $\epsilon$ is related to $c(12)$ by a simple expression ${ }^{15}$ previously thought to be valid only for axisymmetric molecules. ${ }^{12,16}$ 
This apparent contradiction is resolved by observing that in the present development, $c(12)$ is defined with reference to the contracted molecular configuration space $(\mathbf{r}, \mu)$, rather than the complete space of all molecular coordinates. The contracted configuration does not include the angle of rotation about the dipole axis, and thus differs from the full configuration even for rigid molecules. The expression in question, therefore, is indeed valid for rigid polar molecules of arbitrary symmetry, provided that the appropriate definition of $c(12)$ is adopted.

\section{FUNCTIONAL-DERIVATIVE RELATIONS}

Consider a finite volume $V$, of arbitrary shape, containing $N$ identical classical deformable molecules, of arbitrary symmetry and internal structure, at absolute temperature $T$. The number density $N / V$ is denoted by $\rho$. The position and dipole moment vectors of molecule $k$ are denoted by $\mathbf{r}_{k}$ and $\mu_{k}$, respectively, and are collectively represented by the shorthand notation $(k)$.

The single-molecule distribution in $\mathbf{r}$ and $\mu$ is given by

$$
n(\mathbf{r}, \mu)=\left\langle\sum_{k} \delta\left(\mathbf{r}-\mathbf{r}_{k}\right) \delta\left(\mu-\mu_{k}\right)\right\rangle,
$$

where $\delta(x)$ is the three-dimensional Dirac delta function, and the angular brackets $\langle\cdot\rangle\rangle$ signify a canonical ensemble average under the conditions of interest. In the absence of external fields, the fluid will be spatially uniform (except in a negligibly thin surface layer), so that $n(r, \mu)$ will be independent of $r$. Spatial integration of $\mathrm{Eq}$. (1) over the volume $V$ then yields

$$
n(\mathbf{r}, \mu)=\rho s(\mu) \text {, }
$$

where

$$
s(\mu)=\left\langle\delta\left(\mu-\mu_{1}\right)\right\rangle_{0}
$$

is the singlet dipole moment distribution, ${ }^{6}$ and the subscript zero indicates that the average is to be taken in the unperturbed fluid. We note that $\int d \mu s(\mu)=1$.

Since the unperturbed fluid is unpolarized, $s(\mu)$ depends on $\mu$ only through $|\mu|$; it may therefore be written as $s(\mu)$, where $\mu=|\mu|$. It is also convenient to introduce the reduced distribution in $\mu$ alone, call it $p(\mu)$. Clearly

$$
p(\mu)=\int d \mu^{\prime} s\left(\mu^{\prime}\right) \delta\left(\mu-\mu^{\prime}\right)=4 \pi \mu^{2} s(\mu) .
$$

Now suppose that the system is subjected to a singlemolecule external potential $\phi(\mathbf{r}, \mu)$, the effect of which is to add a term $\Sigma_{k} \phi(k)$ to the total potential energy of the system. The single-molecule distribution $n(1)$ then becomes a functional of $\phi(1)$, and is no longer independent of $r_{1}$ and the direction of $\mu_{1}$. Consider the change $\delta n(1)$ in $n(1)$ which accompanies an infinitesimal change $\delta \phi(1)$ in $\phi(1)$. These changes are related by straightforward generalizations of the canonical functional-derivative relations previously developed for rigid mole cules. ${ }^{17}$ One readily verifies that the required generalizations are in fact identical in for $m$ to the original relations; ${ }^{17}$ it is merely necessary to replace the molecular orientation $\omega_{k}$ by $\mu_{k}$ wherever it appears, and hence to interpret the notation $(k)$ in the generalized sense $\left(r_{k}, \mu_{k}\right)$ of the present article. The desired functional-derivative relations are therefore

$$
\begin{aligned}
& \delta n(1)=-\beta \int d(2)[n(1) n(2) h(12)+n(1) \delta(12)][\delta \phi(2)-\delta g], \\
& -\beta \delta \phi(1)+\beta \delta g=\int d(2)[-c(12)+\delta(12) / n(1)] \delta n(2),
\end{aligned}
$$

where $h(12)=h\left(\mathbf{r}_{1}, \mu_{1} ; \mathbf{r}_{2}, \mu_{2}\right)$ is the total correlation function (with the finite-volume correction term subtracted out $\left.^{17}\right), c(12)=c\left(\mathbf{r}_{1}, \mu_{1} ; \mathrm{r}_{2}, \mu_{2}\right)$ is the direct correlation function, $\delta(12)=\delta\left(\mathbf{r}_{1}-\mathbf{r}_{2}\right) \delta\left(\mu_{1}-\mu_{2}\right), g$ is the chemical potential, and $\beta=1 / k T$. The direct and total correlation functions are related by the Ornstein-Zernike equation

$$
h(12)=c(12)+\int d(3) n(3) c(13) h(32)
$$

and the chemical potential variation is given by ${ }^{17}$

$$
\delta g=\int d(1) \delta \phi(1) \partial n(1) / \partial N .
$$

It is well to emphasize that in the present development, the notation $(k)$ refers to a reduced or contracted single-molecule configuration, rather than the complete single-molecule configuration consisting of all the coordinates of molecule $k$. That is to say, in general, there will be other molecular coordinates besides those contained in $\mathbf{r}_{k}$ and $\mu_{k}$. Since the quantities needed to describe dielectric behavior do not directly involve these additional coordinates, the present identification of $(k)$ with $\left(\mathbf{r}_{k}, \mu_{k}\right)$ is appropriate for the purposes of dielectric theory. One must realize, however, that once this identification is adopted, the direct correlation function $c(12)=c\left(\mathbf{r}_{1}, \mu_{1} ; \mathbf{r}_{2}, \mu_{2}\right)$ also relates specifically to the reduced configuration $(\mathbf{r}, \mu)$, and differs from the $c(12)$ that would arise if $(k)$ represented all the coordinates of molecule $k$.

The above relations apply to functional variations about a reference state of arbitrary $\phi(1)$. For present purposes, the appropriate reference state is that with $\phi(1)=0$ (i.e., the unperturbed fluid). Henceforth, this choice will be understood. The reference-state functions $n(1), h(12)$, and $c(12)$ then assume their unper turbed or zero-field forms. Furthermore, we may then regard $\delta \phi(1)$ itself as a weak external potential, and $\delta n(1)$ as the corresponding linear (first-order) deviation of $n(1)$ from its unperturbed value $\rho s\left(\mu_{1}\right)$. All of these interpretations will be understood in what follows.

Since we are concerned with a finite system, it is clear that the spatial integrals in Eqs. (5)-(8) extend only over the volume $V$, even though this is not explicitly indicated by the notation.

To forestall any possible confusion, we remark that the present definition of $h(12)+1$ [see Eq. (5) of Ref. 17] differs from that of Refs. 6 and 10 [see Eq. (2.6) of Ref. 6, from which a factor of 2 is missing on the right side] by a factor of $s\left(\mu_{1}\right) s\left(\mu_{2}\right)$. The present definition adheres more closely to established conventions.

\section{EXISTENCE OF $\epsilon$}

The relations of the preceding section can now be used to determine the static linear response of the polariza- 
tion $\mathbf{P}(\mathbf{r})$, given by

$$
\mathbf{P}(\mathbf{r})=\int d \mu \mu n(\mathbf{r}, \mu)=\int d \mu \mu \delta n(\mathbf{r}, \mu),
$$

to a weak external electric field $E_{0}(r)$. It is assumed that $E_{0}(r)$ varies slowly, in a molecular sense, with the position $\mathbf{r}$. The perturbation potential corresponding to $\mathbf{E}_{0}(\mathbf{r})$ is given by

$$
\delta \phi(1)=-\mu_{1} \cdot E_{0}\left(r_{1}\right) \text {. }
$$

Combining Eqs. (2), (8), and (10), we find that $\delta g=0$ for this potential, so the $\delta g$ terms in Eqs. (5) and (6) may henceforth be omitted. Equation (6) therefore becomes

$$
\beta \mu_{1} \cdot \mathbf{E}_{0}\left(\mathbf{r}_{1}\right)=\int d(2)[-c(12)+\delta(12) / n(1)] \delta n(2) .
$$

Sufficient conditions for the existence of $\epsilon$ are most easily expressed in terms of the behavior of $c(12)$. We adopt the fundamental assumption that $c(12)$ is of the form

$$
c(12)=c_{0}(12)-\beta \theta(12),
$$

where $c_{0}(12)$ is a short-ranged function (i.e., it goes to zero faster than $\left|\mathbf{r}_{1}-\mathbf{r}_{2}\right|^{-3}$ ) which depends only on relative positions and dipole moment directions in addition to $\mu_{1}$ and $\mu_{2}$ (i。e $e_{o}$, it is translationally and rotationally invariant). The function $\theta(12)$ is the dipole-dipole potential with a spherical cutoff:

$$
\begin{aligned}
& \theta(12)=-\mu_{1} \cdot \mathbf{T}_{0}\left(\mathbf{r}_{1}-\mathbf{r}_{2}\right) \cdot \mu_{2}, \\
& \mathbf{T}_{0}(\mathbf{r})=H(|\mathbf{r}|-\sigma) \nabla \nabla|\mathbf{r}|^{-1},
\end{aligned}
$$

where $H(x)$ is the Heaviside unit step function $[H(x)$ is unity for $x \geq 0$ and zero otherwise], and it is understood that the limit $\sigma \rightarrow 0$ is ultimately to be taken.

We now proceed to show that the above assumption implies the existence of the dielectric constant $\epsilon$; i.e., that the conditions embodied in Eq. (12) are sufficient for the existence of $\epsilon$. This is not surprising, as these conditions are closely analogous to the known sufficient conditions for rigid polar molecules. ${ }^{12,14}$ It seems very likely that Eq. (12) will in fact ordinarily be satisfied (except of course in a negligibly thin surface layer), but this is a separate question that will not be pursued here.

Combining Eqs. (11)-(13), we obtain

$$
\beta \mu_{1} \cdot \mathbf{E}_{L}\left(\mathbf{r}_{1}\right)=\int d(2)\left[-c_{0}(12)+\delta(12) / n(1)\right] \delta n(2),
$$

where

$$
\mathbf{E}_{L}(\mathbf{r})=\mathbf{E}_{0}(\mathbf{r})+\int d \mathbf{r}^{\prime} \mathbf{T}_{0}\left(\mathbf{r}-\mathbf{r}^{\prime}\right) \cdot \mathbf{P}\left(\mathbf{r}^{\prime}\right)
$$

is the Lorentz electric field, which is related to the Maxwell electric field $E(\mathbf{r})$ by $\mathbf{E}_{L}(\mathbf{r})=\mathbf{E}(\mathbf{r})+(4 \pi / 3) \mathbf{P}(\mathbf{r})$. Equation (15) may be inverted by introducing a shortranged total correlation function $h_{0}(12)$ defined by

$$
h_{0}(12)=c_{0}(12)+\int d(3) n(3) c_{0}(13) h_{0}(32) \text {. }
$$

We then obtain

$$
\delta n(1)=\beta \int d(2)\left[n(1) n(2) h_{0}(12)+n(1) \delta(12)\right] \mu_{2} \cdot \mathbf{E}_{L}\left(\mathbf{r}_{2}\right)
$$

Since $c_{0}(12)$ is short-ranged, so is $h_{0}(12)$. The spatial integrals in Eqs. (15), (17), and (18) may therefore be extended over all space instead of just over the volume $V$.

Since $\mathbf{E}_{0}(\mathbf{r})$ is slowly varying and the system is a fluid, it is clear that $P(r)$ and hence $E_{L}(r)$ will also be slowly varying. We may therefore evaluate $E_{L}\left(r_{2}\right)$ in Eq. (18) at the point $\mathbf{r}_{2}=\mathbf{r}_{1}$ and take it outside the integral over $\mathbf{r}_{2}$. When this is done, the result combines with Eq. (9) to yield

$$
\mathbf{P}(\mathbf{r})=\mathbf{A}(\mathbf{r}) \cdot \mathbf{E}_{L}(\mathbf{r}),
$$

where

$\mathbf{A}\left(\mathbf{r}_{1}\right)=\beta \rho \int d \mathbf{r}_{2} d \mu_{1} d \mu_{2} s\left(\mu_{1}\right)\left[\rho s\left(\mu_{2}\right) h_{0}(12)+\delta(12)\right] \mu_{1} \mu_{2}$,

and use has been made of Eq. (2). But since $c_{0}(12)$ is translationally and rotationally invariant, the same is clearly true for $h_{0}(12)$. Therefore $\mathbf{A}(\mathbf{r})$ is independent of $\mathbf{r}$ and proportional to the unit dyadic $\mathbf{U}$. That is, $\mathbf{A}(\mathbf{r})=A \mathbf{U}$, where $A=(1 / 3) \mathbf{A}: \mathbf{U}$. Equation (19) now reduces to $\mathrm{P}(\mathrm{r})=A \mathrm{E}_{L}(\mathrm{r})$, which show $\mathrm{s}^{15}$ that $\epsilon$ exists and is given by $(\epsilon-1) /(\epsilon+2)=4 \pi A / 3$; i.e.,

$\frac{\epsilon-1}{\epsilon+2}=\frac{4 \pi}{9} \beta \rho \int d \mathrm{r}_{2} d \mu_{1} d \mu_{2} s\left(\mu_{1}\right)\left[\rho s\left(\mu_{2}\right) h_{0}(12)+\delta(12)\right] \mu_{1} \cdot \mu_{2}$

We have therefore shown that Eq. (12) implies the existence of $\epsilon$, with $\epsilon$ given by Eq. (21). This expression for $\epsilon$ does not appear to have been previously presented, although it is closely related to the known expression for $\epsilon$ in fluid mixtures of rigid polar molecules. ${ }^{13,14}$ Such a relation is not unexpected in view of the correspondence between fluids of deformable molecules and fluid mixtures of rigid molecules. ${ }^{8,9}$ The relation becomes clearer when $\mathrm{Eq}$. (21) is rewritten in the equivalent form

$\frac{\epsilon-1}{\epsilon+2}=\frac{4 \pi}{9} \beta \rho \int d \mu d \mu^{\prime} p(\mu)\left[\delta\left(\mu-\mu^{\prime}\right)+\rho p\left(\mu^{\prime}\right) H\left(\mu, \mu^{\prime}\right)\right] \mu \mu^{\prime}$,

where

$$
\begin{aligned}
H\left(\mu, \mu^{\prime}\right)= & (4 \pi)^{-2}\left(\mu \mu^{\prime}\right)^{-3} \int d \mathbf{r}_{2} d \mu_{1} d \mu_{2} \delta\left(\mu-\mu_{1}\right) \\
& \times \delta\left(\mu^{\prime}-\mu_{2}\right) h_{0}(12) \mu_{1} \cdot \mu_{2}
\end{aligned}
$$

Since $\rho p(\mu) d \mu$ is just the partial number density of molecules with $|\mu|$ in the interval $(\mu, \mu+d \mu)$, the structural resemblance of $\mathrm{Eq}$. (22) to the corresponding for mula for rigid-dipole mixtures [see Eq. (17) of Ref. 14] is obvious.

Although we have not found it convenient to do so, the delta-function terms in Eqs. (21) and (22) can be trivially evaluated in terms of the quantity $\int d \mu \mu^{2} s(\mu)$ $=\int d \mu \mu^{2} p(\mu)$, which is just the mean square dipole moment of an individual molecule. If desired, this quantity can then be rewritten in terms of an effective permanent dipole moment and polarizability, ${ }^{5}$ but such a representation has little apparent advantage. 


\section{IV. $\epsilon$ IN TERMS OF $c(12)$}

In this section we derive the expression for $\epsilon$ in terms of $c(12)$. We first multiply Eq. (17) by $(4 \pi)^{-2}\left(\mu \mu^{\prime}\right)^{-3}$ $\times \delta\left(\mu-\mu_{1}\right) \delta\left(\mu^{\prime}-\mu_{2}\right) \mu_{1} \cdot \mu_{2}$ and integrate over $\mathbf{r}_{2}, \mu_{1}$, and $\mu_{2}$. This yields

$$
\begin{aligned}
H\left(\mu, \mu^{\prime}\right)= & C\left(\mu, \mu^{\prime}\right)+(4 \pi)^{-2}\left(\mu \mu^{\prime}\right)^{-3} \rho \int d \mu_{1} d(2) d(3) \\
& \times s\left(\mu_{3}\right) \delta\left(\mu-\mu_{1}\right) \delta\left(\mu^{\prime}-\mu_{2}\right) c_{0}(13) h_{0}(32) \mu_{1} \cdot \mu_{2},
\end{aligned}
$$

where

$$
\begin{aligned}
C\left(\mu, \mu^{\prime}\right)= & (4 \pi)^{-2}\left(\mu \mu^{\prime}\right)^{-3} \int d \mathbf{r}_{2} d \mu_{1} d \mu_{2} \\
& \times \delta\left(\mu-\mu_{1}\right) \delta\left(\mu^{\prime}-\mu_{2}\right) c_{0}(12) \mu_{1} \cdot \mu_{2} \\
= & (4 \pi)^{-2}\left(\mu \mu^{\prime}\right)^{-3} \int d \mathbf{r}_{2} d \mu_{1} d \mu_{2} \\
& \times \delta\left(\mu-\mu_{1}\right) \delta\left(\mu^{\prime}-\mu_{2}\right) c(12) \mu_{1} \cdot \mu_{2} .
\end{aligned}
$$

The second equality in Eq. (25) follows easily from Eqs, (12) and (13), together with the fact ${ }^{12}$ that $\mathbf{U}: \mathbf{T}_{0}(\mathbf{r})=0$. We note parenthetically that both $H\left(\mu, \mu^{\prime}\right)$ and $C\left(\mu, \mu^{\prime}\right)$ are symmetric in $\mu$ and $\mu^{\prime}$.

Since $h_{0}(12)$ is translationally and rotationally invar iant, it is clear that

$$
\int d(2) \delta\left(\mu^{\prime}-\mu_{2}\right) h_{0}(32) \mu_{2}=f\left(\mu_{3}, \mu^{\prime}\right) \mu_{3},
$$

where $f\left(\mu_{3}, \mu^{\prime}\right)$ is independent of $r_{3}$. To determine the form of $f\left(\mu, \mu^{\prime}\right)$, we take the dot product of $\mathrm{Eq}$. (26) with $\mu_{3}$, multiply by $\delta\left(\mu-\mu_{3}\right)$, and integrate over $\mu_{3}$. We thereby obtain

$$
f\left(\mu, \mu^{\prime}\right)=4 \pi \mu^{2}\left(\mu^{\prime} / \mu\right)^{3} H\left(\mu, \mu^{\prime}\right) \text {. }
$$

Equations (24)-(27) now combine to yield

$$
H\left(\mu, \mu^{\prime}\right)=C\left(\mu, \mu^{\prime}\right)+\rho \int d \mu_{1} p\left(\mu_{1}\right) C\left(\mu, \mu_{1}\right) H\left(\mu_{1}, \mu^{\prime}\right) .
$$

One readily verifies that $\mathrm{Eq} .(28)$ is equivalent to

$$
\int d \mu_{1} L\left(\mu, \mu_{1}\right) K\left(\mu_{1}, \mu^{\prime}\right)=\delta\left(\mu-\mu^{\prime}\right)
$$

where

$$
\begin{aligned}
& K\left(\mu, \mu^{\prime}\right)=\rho p(\mu)\left[\delta\left(\mu-\mu^{\prime}\right)+\rho p\left(\mu^{\prime}\right) H\left(\mu, \mu^{\prime}\right)\right], \\
& L\left(\mu, \mu^{\prime}\right)=-C\left(\mu, \mu^{\prime}\right)+\delta\left(\mu-\mu^{\prime}\right) /[\rho p(\mu)] .
\end{aligned}
$$

But $K\left(\mu, \mu^{\prime}\right)$ is just the kernel that appears in $\mathrm{Eq}$. (22) for $\epsilon$. The expression for $\epsilon$ in terms of $c(12)$ is therefore

$$
\frac{\epsilon-1}{\epsilon+2}=\frac{4 \pi}{9} \beta \int d \mu d \mu^{\prime} K\left(\mu, \mu^{\prime}\right) \mu \mu^{\prime},
$$

where $K\left(\mu, \mu^{\prime}\right)$ is now regarded as the inverse kernel defined by Eq. (29), which is related to $C\left(\mu, \mu^{\prime}\right)$ by Eq. (31) and thence to $c(12)$ via Eq. (25).

\section{SPECIALIZATION TO RIGID MOLECULES}

It is instructive to specialize the present results to the case of rigid polar molecules, to which they lend some new insight. In this special case we have

$$
p(\mu)=\delta\left(\mu-\mu_{0}\right),
$$

where $\mu_{0}$ is the fixed value of the molecular dipole moment. Since $\mu_{k}=\mu_{0}$ for all $k$ at all times, the functions $h(12), c(12), h_{0}(12)$, and $c_{0}(12)$ now depend only on $\left(\mathbf{r}_{1}, \boldsymbol{\omega}_{1} ; \mathbf{r}_{2}, \omega_{2}\right)$, where $\omega_{k}=\left(\theta_{k}, \phi_{k}\right)$ represents the polar and azimuthal angles that specify the direction of $\mu_{k}$. Now $d \mu_{k}=\mu_{k}^{2} d \mu_{k} d \omega_{k}$, where $d \boldsymbol{\omega}_{k}=\sin \theta_{k} d \theta_{k} d \phi_{k}$. The Ornstein-Zernike equation [Eq. (7)] therefore reduces to

$$
h(12)=c(12)+(\rho / 4 \pi) \int d \mathbf{r}_{3} d \boldsymbol{\omega}_{3} c(13) h(32),
$$

where use has been made of Eqs. (2), (4), and (33). [The same reduction, namely $\int d(3) n(3) \rightarrow(\rho / 4 \pi) \int d \mathbf{r}_{3} d \boldsymbol{\omega}_{3}$, of course applies to Eq. (17) as well.] We see that Eq. (34) is formally the same as the Ornstein-Zernike equation for rigid axisymmetric molecules, ${ }^{15}$ even though we have not restricted the molecular symmetry in any way. The reason for this correspondence is that the angle $\psi_{k}$ of rotation about the axis defined by $\omega_{k}$ is not included in the present reduced description. This angle is therefore averaged over in the definitions of $n(1)$ and $h(12)$, so that the molecules may be regarded as axisymmetric "on the average." It may be helpful to think of $h(12)$ and $c(12)$ as the correlation functions for a hypothetical system of equivalent axisymmetric molecules, but such an interpretation is in no way essential.

By virtue of Eq. (33), Eq. (22) for $\epsilon$ now becomes

$$
(\epsilon-1) /(\epsilon+2)=y\left(1+\rho H_{0}\right) \text {, }
$$

where $y=(4 \pi / 9) \beta \rho \mu_{0}^{2}$ and $H_{0}=H\left(\mu_{0}, \mu_{0}\right)$. From Eq. (23) we find that

$$
H_{0}=(4 \pi)^{-2} \int d \mathbf{r}_{2} d \omega_{1} d \omega_{2} h_{0}(12) \mathbf{e}_{1} \cdot \mathbf{e}_{2},
$$

where $\mathrm{e}_{k}$ is the unit vector with orientation $\omega_{k}$; i.e., $\mathbf{e}_{k}=\mu_{k} / \mu_{0}$. Similarly, we find from Eq. (25) that

$$
C_{0}=(4 \pi)^{-2} \int d \mathbf{r}_{2} d \boldsymbol{\omega}_{1} d \boldsymbol{\omega}_{2} c(12) \mathbf{e}_{1} \cdot \mathbf{e}_{2},
$$

where $C_{0}=C\left(\mu_{0}, \mu_{0}\right)$. But according to Eqs. (28) and (33), $H_{0}$ and $C_{0}$ are related by

$$
H_{0}=C_{0}+\rho C_{0} H_{0},
$$

from which it follows that $\left(1+\rho H_{0}\right)=\left(1-\rho C_{0}\right)^{-1}$. Equation (35) may therefore be written in the alternative form

$$
(\epsilon-1) /(\epsilon+2)=\nu\left(1-\rho C_{0}\right)^{-1} \text {. }
$$

We now observe that Eq. (39) is formally identical to an earlier result ${ }^{15}$ previously thought to be valid only for axially symmetric molecules. ${ }^{12,16}$ This expression for $\epsilon$ is therefore actually valid more generally; it applies to rigid polar molecules of arbitrary symmetry, provided that $c(12)$ is properly interpreted. That is, $c(12)$ must be regarded as the function of $\left(\mathbf{r}_{1}, \boldsymbol{\omega}_{1} ; \mathbf{r}_{2}, \omega_{2}\right)$ defined by Eq. (34), in which $h(12)$ represents the total correlation function in the variables $\left(\mathbf{r}_{1}, \omega_{1} ; \mathbf{r}_{2}, \boldsymbol{\omega}_{2}\right)$ alone without regard to the angles $\psi_{1}$ and $\psi_{2}$. Confusion with the more conventional $c(12)$ for rigid molecules, ${ }^{12}$ which results when $(k)$ is used to represent the complete single-molecule configuration $\left(\mathbf{r}_{k}, \omega_{k}, \psi_{k}\right)$, can be avoided by paying close attention to the function arguments. 
${ }^{1}$ J. M. Deutch, Annu. Rev. Phys. Chem. 24, 301 (1973).

${ }^{2}$ S. A. Adelman and J. M. Deutch, Adv. Chem. Phys. 31, 103 (1975).

${ }^{3}$ M. S. Wertheim, Annu. Rev. Phys. Chem, 30, 471 (1979).

${ }^{4}$ G. Stell, G. N. Patey, and J. S. Hóye, Adv. Chem. Phys. 48,183 (1981).

${ }^{5}$ D. Chandler, J. Chem. Phys. 67, 1113 (1977).

${ }^{6}$ L. R. Pratt, Mol. Phys, 40, 347 (1980).

7J. S. Høye and G. Stell, J. Chem. Phys. 72, 1597 (1980).

${ }^{8}$ J. S. Hoye and G. Stell, J. Chem. Phys. 73, 461 (1980).

${ }^{9}$ J. S. Høye and G. Stell, J. Chem. Phys. 75, 3565 (1981).
${ }^{10} \mathrm{~J}$. D. Ramshaw, J. Chem. Phys. (in press).

${ }^{11}$ W. F. Brown, Jr., in Handbuch der Physik, edited by S. Flügge (Springer, Berlin, 1956), Vol. 17, p. 1.

${ }^{12} \mathrm{~J}$. D. Ramshaw, J. Chem. Phys, 68, 5199 (1978), and references cited therein.

${ }^{13}$ J. S. Hoye and G. Stell, J. Chem. Phys. 70, 2894 (1979).

${ }^{14} \mathrm{~J}$. D. Ramshaw and N. D. Hamer, J. Chem. Phys. 75, 3511 (1981).

${ }^{15}$ J. D. Ramshaw, J. Chem. Phys, 57, 2684 (1972).

${ }^{16}$ J. S. Hoye and G. Stell, J. Chem. Phys. 61, 562 (1974).

${ }^{17}$ J. D. Ramshaw, Mol. Phys. 41, 219 (1980). 